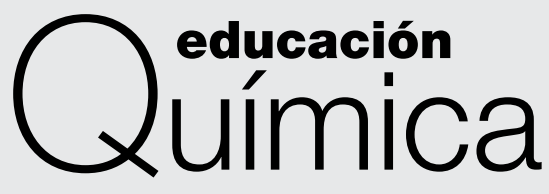

www.educacionquimica.info

\title{
INVESTIGACIÓN EDUCATIVA
}

\section{Coherencia y congruencia en las representaciones utilizadas por estudiantes universitarios acerca de los estados de agregación}

\author{
Osvaldo Cappannini ${ }^{\mathrm{a}, \mathrm{b}, *}$ y Carlos Espíndola ${ }^{\mathrm{b}, \mathrm{c}}$ \\ a Facultad de Ciencias Exactas, Universidad Nacional de La Plata, Buenos Aires, Argentina

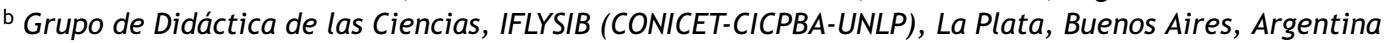 \\ c Facultad de Humanidades y Ciencias de la Educación, Universidad Nacional de La Plata, Buenos Aires, Argentina
}

Recibido el 25 de marzo de 2017; aceptado el 28 de marzo de 2017

Disponible en Internet el 13 de mayo de 2017

\section{PALABRAS CLAVE \\ Representaciones; \\ Estructura de la \\ materia; \\ Modelo corpuscular}

\section{KEYWORDS}

Representations; Structure of matter; Corpuscular model

\begin{abstract}
Resumen Existe una extensa lista de trabajos de investigación dedicados a identificar representaciones sobre estructura de la materia en personas pertenecientes a diferentes niveles en el sistema educativo. Uno de los objetivos de la educación, tanto secundaria como universitaria, es que los estudiantes aprendan a interpretar fenómenos macroscópicos en términos de modelos discretos y microscópicos. El modelo corpuscular resulta fundamental para poder explicar, por ejemplo, las diferencias entre estados de agregación de la materia, sus propiedades y los cambios, físicos o químicos, que experimentan. Las investigaciones realizadas muestran que los estudiantes aceptan fácilmente este modelo corpuscular y parte de la terminología que incluye, pero que no lo utilizan espontáneamente. En este trabajo se presenta una indagación sobre representaciones de estructura para los 3 estados de agregación en estudiantes universitarios y un análisis de la utilización conjunta de modelos, reflejando que también en este nivel, y para estudiantes de cursos introductorios de Química, el modelo discreto de la materia aceptado por la comunidad científica solo resulta dominante para gases pero no para sólidos y líquidos.

() 2017 Universidad Nacional Autónoma de México, Facultad de Química. Este es un artículo Open Access bajo la licencia CC BY-NC-ND (http://creativecommons.org/licenses/by-nc-nd/4.0/).
\end{abstract}

Coherence and congruence in the representations used by university students about the states of aggregation

Abstract We can find an extensive list of research articles aimed to identify structure of matter representations in people belonging to several levels in the educative system. Students' learning of the explanation of macroscopic phenomena in terms of discrete and microscopic models constitutes one of the purposes of secondary and university education. The corpuscular model

\footnotetext{
* Autor para correspondencia.

Correo electrónico: cappa@iflysib.unlp.edu.ar (0. Cappannini).

La revisión por pares es responsabilidad de la Universidad Nacional Autónoma de México.
} 
is essential to explain, e.g., the differences among the three states of matter, their properties and the (physical or chemical) changes they may undergo. Research has shown that although students easily accept this corpuscular model and some of its terminology, they do not use it spontaneously. This article presents an inquiry on representations about structure of the three states of matter in university students together with an evaluation of their use for combinations of states, showing that also in this level and for students belonging to Introductory Chemistry courses, the discrete model of matter accepted by the scientific community is dominant only for gases but not for solids and liquids.

(c) 2017 Universidad Nacional Autónoma de México, Facultad de Química. This is an open access article under the CC BY-NC-ND license (http://creativecommons.org/licenses/by-nc-nd/4.0/).

\section{Introducción}

Se ha señalado (Linder y Marshall, 2003) que cada individuo aborda los problemas, en distintos temas y contextos, utilizando representaciones personales al interpretar situaciones y fenómenos. Desde esta perspectiva, no se pueden considerar unas representaciones mejores que otras, sino que hay modelos más o menos adecuados al contexto en el que se van a utilizar. Asimismo, el término representación implica una construcción mental en la que confluyen los conocimientos previos y los recursos cognitivos disponibles además de las características identificadas en la situación que se enfrenta (Hammer, 2004). Las representaciones en los estudiantes, de naturaleza cognitiva, resultan de la interacción con su entorno, en el marco de su relación con la institución educativa y el ámbito social a través de vínculos con pares y docentes (Pozo y Rodrigo, 2001; Taber y García-Franco, 2010). El estudio de la construcción y utilización en diferentes contextos de estas representaciones aporta a la comprensión de los procesos cognitivos y puede brindar información acerca del marco conceptual del estudiante, es decir, de su estructura subyacente de conocimientos (Norman, 1983; Johnson-Laird, 1983). Así, el análisis de modelos sobre estructura de la materia presentes en estudiantes de diferentes niveles del sistema educativo se han constituido en tema de investigación continuo (Krnel, Watson y Glazar, 2005; Stains y Talanquer, 2007). Por otra parte, las respuestas de la ciencia a cuestiones vinculadas a propiedades de sustancias diversas, constituidas en teorías y modelos, forman un cuerpo de conocimientos que, mediados por la transposición didáctica, se recogen organizados en los currículos educativos. De una adecuada articulación entre ellos y las representaciones presentes en cada estudiante, dependerá el proceso de aprendizaje (FloresCamacho, Gallegos-Cázares, Garritz y García-Franco, 2007; Cooper, Corley y Underwood, 2013).

Una presunción habitual es que los ingresantes al nivel universitario conocen la denominada teoría de partículas, uno de los núcleos de la formación en ciencias en el nivel secundario. El modelo que propone a la materia constituida por partículas en continuo movimiento separadas por vacío permite explicar muchas propiedades macroscópicas. Habitualmente esta teoría se presenta como una serie de afirmaciones y dibujos (Giudice y Galagovsky, 2008), aplicadas a, por ejemplo, cambios de estados de agregación.
Existe abundante bibliografía ilustrativa de dificultades que surgen al interpretar fenómenos químicos usando modelos discretos, por ejemplo, asignar características macroscópicas a las partículas constituyentes de las sustancias $u$ otras que surgirían de la interacción entre lo que los estudiantes ya conocen y lo propuesto desde el conocimiento escolar (García-Franco y Taber, 2010; Taber y García-Franco, 2010; Adbo y Taber, 2014). Resulta por demás importante conocer si, luego de cursos de Química universitarios, los estudiantes han incorporado adecuadamente el modelo discreto de la materia reflejado en su explicación de propiedades macroscópicas de materiales y si utilizan un único modelo para diferentes estados de agregación y distintos fenómenos. El reconocimiento de estas cuestiones podría llevar a modificaciones en las actividades de aula relacionadas con uno de los lados del triángulo de Johnstone (Johnstone, 2010), aquel que vincula los vértices macroscópico y submicroscópico y que, mediante modelizaciones, accede a explicaciones disciplinares sobre problemáticas del mundo natural.

El universo analizado incluyó 378 estudiantes del ciclo básico de varias carreras de la Facultad de Ciencias Exactas, Universidad Nacional de La Plata (UNLP) (293 finalizando el curso 2007 de Química General y 85 cursando Física I del 2013, posterior al anterior), y 69 estudiantes concluyendo la materia Química General del primer año de la Licenciatura en Alimentos de la Universidad Nacional del Noroeste de la Provincia de Buenos Aires (UNNOBA). Los cursos de Química General incluían recorridos tradicionales basados en textos habituales (Chang, 1995; Atkins y Jones, 1998; Glasstone, 1970).

\section{Metodología e instrumento utilizado}

La encuesta utilizada, individual y anónima, abarcó cuestiones que proponen interpretar la compresión de sustancias en las 3 fases desde un modelo discreto de la materia (sin referencia al movimiento de las partículas y sin deslindar el contenido de este término) o una mirada estrictamente macroscópica (tabla 1). Mediante ella se buscó identificar las representaciones utilizadas para las fases; caracterizar el uso de una misma representación en forma congruente para diferentes estados de agregación y registrar si las tendencias observadas en UNNOBA 2007 (Espíndola y Cappannini, 2012) se mantenían en otras instituciones (como UNLP) y en 
Tabla 1 Encuesta utilizada para evaluar representaciones de estudiantes universitarios sobre diferentes estados de agregación

Responder llenando la planilla de respuestas adjunta. En caso de elegir la opción «ninguna de las anteriores» explicar brevemente la opinión personal en el reverso de la planilla para respuestas

1) Considera una jeringa con aire, que tiene tapada la salida. Si empujas el émbolo podrás desplazarlo hasta cierto punto haciendo que el espacio ocupado por el aire disminuya. ¿Qué te parece que sucede? Responde eligiendo una o más de las siguientes posibles respuestas:

a) Disminuye el volumen de aire

b) Disminuye el volumen de las partículas que lo forman

c) Disminuye el espacio entre las partículas que lo forman d) No cambia el volumen de aire

e) No cambia el volumen de las partículas que lo forman

f) No cambia el espacio entre las partículas que lo forman g) Ninguna de las anteriores

2) Considera una jeringa con agua, que tiene tapada la salida. Si empujas el émbolo podrás desplazarlo, con algún esfuerzo, hasta cierto punto haciendo que el espacio ocupado por el agua disminuya. ¿Qué te parece que sucede? Responde eligiendo una o más de las siguientes posibles respuestas:

a) Disminuye el volumen de agua

b) Disminuye el volumen de las partículas que la forman

c) Disminuye el espacio entre las partículas que la forman

d) No cambia el volumen de agua

e) No cambia el volumen de las partículas que la forman

f) No cambia el espacio entre las partículas que la forman

g) Ninguna de las anteriores

3) Considera un recipiente cilíndrico rígido lleno de plastilina y provisto de un pistón en la parte superior. Mediante un dispositivo de alta presión se empuja el pistón hacia abajo haciendo que el espacio ocupado por la plastilina

disminuya. ¿Qué te parece que sucede? Responde eligiendo una o más de las siguientes posibles respuestas:

a) Disminuye el volumen de plastilina

b) Disminuye el volumen de las partículas que la forman

c) Disminuye el espacio entre las partículas que la forman

d) No cambia el volumen de plastilina

e) No cambia el volumen de las partículas que la forman

f) No cambia el espacio entre las partículas que la forman g) Ninguna de las anteriores

cohortes diferentes. Las encuestas se realizaron en clase, con la presencia de los docentes a cargo de los cursos y enmarcándolas en actividades de investigación.

Para superar subjetividades en la consideración de las respuestas obtenidas, Duschl, Hamilton y Grandy (1990) y Taber y Watts (1996) se refieren a imperativos sociales y metafísicos surgiendo de la misma interacción humana ante los requerimientos formulados y que los individuos intentan satisfacer de la mejor manera posible. Puede interpretarse así que los encuestados han respondido acudiendo a conocimientos incorporados durante su formación en el sistema educativo, a aquellos elaborados de manera personal o a cubrir la simple necesidad de responder (imperativo social) o explicar (imperativo metafísico).
En el tratamiento de lo obtenido se acudió a criterios de selección y ordenamiento similares a los descriptos en Espíndola y Cappannini (2012), basados en una perspectiva fenomenográfica (Ebenezer y Fraser, 2001) en la que no se establecen categorías a priori, sino que estas se generan agrupando respuestas para caracterizar el espacio de representaciones del colectivo encuestado. Establecer dicho espacio requirió identificar regularidades en un número muy grande de datos, por lo cual se precisó una disposición particular de los mismos. En el análisis realizado se consideró que la opción (a) (ver tabla 1) implica, desde una mirada macroscópica y sin explicitar representación discreta o continua, aceptar la compresión del material, mientras que la (d) niega esta situación. Las restantes opciones (todas referidas al modelo discreto) reflejan una interpretación microscópica del fenómeno.

El proceso de análisis permitió confluir en las categorías mostradas en la tabla 2 de acuerdo con las opciones seleccionadas por los estudiantes. Así las elecciones del conjunto «a-c-e» responderían a una representación discreta (dadas las opciones «c» $\mathrm{y}$ «e») y coherente con lo macroscópico (de acuerdo con la selección de «a»). Aquellas con respuestas «a» junto con «d» implicarían la contradicción de registrar que un cuerpo es comprimido (opción «a») y, a la vez, se niega su compresión (opción «d»).

Las categorías pueden simplificarse notando que, aunque no coincidan las tríadas, pueden coincidir de categoría como se advierte en los casos «d-e-f» $y$ «b-c-d» incluidas en la categoría I (corresponde a una representación discreta pero que no acepta lo observable). Para la primera tríada, «d» significa que no se acepta la compresión, mientras que «e-f» implica una representación discreta en la que ni las partículas ni el espacio entre ellas cambian su volumen, lo cual supone no aceptar lo advertido en el enunciado. También para «b-c-d», «b» $y$ «c» significan que hay una representación discreta (en la que tanto el volumen de las partículas como el espacio entre ellas disminuyen) pero, según «d», no hay compresión.

Las categorías indicadas en la tabla 2 resultaron del conjunto de respuestas obtenidas en las cohortes 2007 de UNLP y UNNOBA, confirmándose en la cohorte 2013 de UNLP (es decir, no aparecieron otras combinaciones de respuestas que las allí mostradas). Además del modelo deseable desde el saber escolar (categoría ॥), se ha identificado uno que incluye «partículas» con características macroscópicas (categoría III). La categoría IV implica una representación discreta contradictoria en lo microscópico (por ejemplo, al incluir las opciones «c» $\mathrm{y}\langle\mathrm{f} »)$, mientras que la categoría $v$ contiene respuestas sin una representación discreta (opciones «a» o «d» solas). Las categorías VI y vII completan las opciones seleccionadas.

\section{Análisis de las respuestas}

\section{Sobre coherencia entre lo observable y los modelos microscópicos}

En la figura 1 se vuelca el porcentaje de respuestas, por categoría y estado de agregación, para la población de estudiantes de la UNLP, curso 2007. Domina la categoría ॥ en los 3 estados de agregación, aunque en gas resulta más notable 
Tabla 2 Categorías que aparecieron en las cohortes encuestadas, su descripción y las respuestas que incluyen

\begin{tabular}{|c|c|c|}
\hline Categoría & Descripción & Respuestas \\
\hline 1 & $\begin{array}{l}\text { Modelo discreto que no refleja coherencia con lo } \\
\text { observable }\end{array}$ & $\begin{array}{l}(a-e-f),(b-d),(b-c-d),(b-d-f),(c-d),(c-d-g), \\
(c-d-e),(e-f),(d-e-f),(d-e),(d-f) \text { y }(d-e-f-g)\end{array}$ \\
\hline II & Modelo discreto coherente con lo macroscópico & $(a-c-e),(a-e),(a-c),(c-e),(c)$ y $(e)$ \\
\hline III & $\begin{array}{l}\text { Modelo discreto que asigna características } \\
\text { macroscópicas a las partículas }\end{array}$ & $(a-b-c),(a-b-f),(b-c),(b),(a-b)$ y $(b-f)$ \\
\hline IV & Modelo discreto pero contradictorio & $\begin{array}{l}(a-b-e),(b-e-f),(a-c-f),(c-d-e-f),(c-d-f) \text { y } \\
(a-c-e-f)\end{array}$ \\
\hline V & No refleja modelo discreto & (d), (d-g) y (a) \\
\hline VI & Desacuerdo con alternativas ofrecidas & (g) \\
\hline Otras & Contradictorias o sin respuesta & (a-d-e-f), (a-d-e), (a-d), NS/NC \\
\hline
\end{tabular}

(82\% frente a $50 \%$ y $46 \%$ en líquido y sólido, respectivamente). Además, se percibe una gran dispersión en otras categorías: la I posee notables porcentajes para los 3 estados de agregación; la III es muy baja para gas y líquido y crece algo para sólido; la v es muy baja para gas, aumenta algo para líquido y aún más para sólido; las restantes categorías solo se registran en líquido y sólido y resultan muy menores. Esta dispersión para líquido y sólido evidenciaría dificultades en el uso del modelo asociado a la teoría de partículas para explicar lo macroscópico, acudiéndose a una diversidad de representaciones distantes del conocimiento científico.

La figura 2 muestra los porcentajes de la cohorte 2007 de UNNOBA. Al comparar las figuras 1 y 2 se observan diferencias de perfiles entre ambas universidades. Las respuestas en UNNOBA aparecen más dispersas que las de UNLP: por ejemplo, la categoría ॥ para UNNOBA solo llega al 45\% para gas, al $26 \%$ para líquido y al $15 \%$ para sólido. En las demás categorías se identifica un perfil análogo. Sin embargo, se evidencian comportamientos similares en los 2 ámbitos: a) la categoría II, mayoritaria para gas, disminuye para líquido y aún más para sólido; b) la categoría ı es la siguiente en importancia y muestra un porcentaje, en UNLP, que asciende de gas a líquido y vuelve a bajar para sólido, mientras que en UNNOBA crece de gas a sólido convirtiéndose en

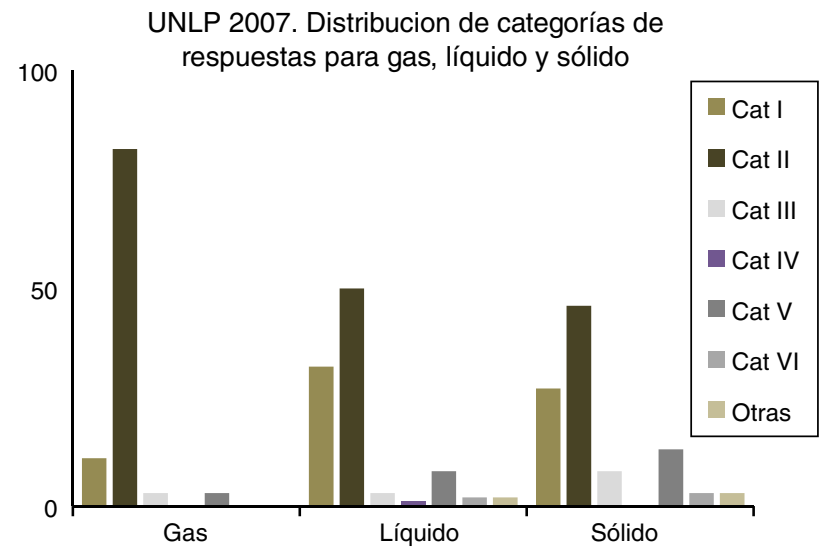

Figura 1 Distribución de respuestas para gas, líquido y sólido en estudiantes de UNLP (Química General, curso 2007). UNLP: Universidad Nacional de La Plata.

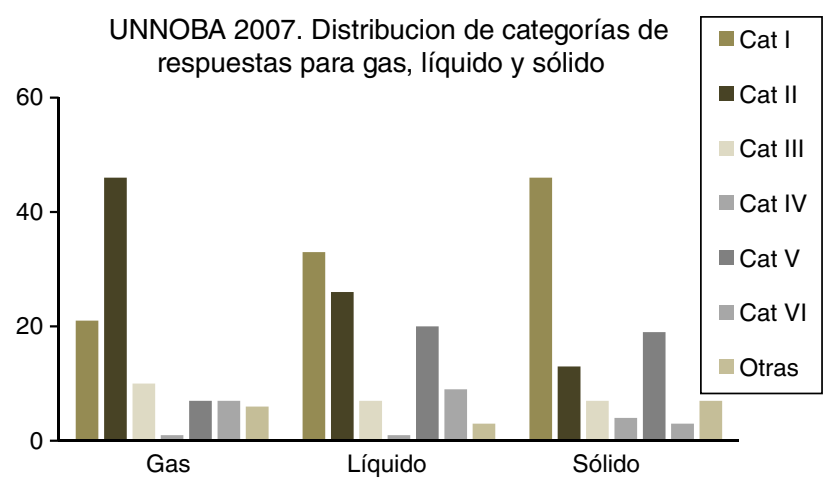

Figura 2 Distribución de respuestas para gas, líquido y sólido en estudiantes de UNNOBA (Química General, curso 2007). UNNOBA: Universidad Nacional del Noroeste de la Provincia de Buenos Aires.

esta última fase en dominante, y c) sigue la categoría v: es baja para gas y notable para líquido y sólido en ambos casos.

En la figura 3 se incluyen los resultados obtenidos para UNLP en 2013 evidenciando un perfil análogo al obtenido en 2007, lo que avalaría, de algún modo, la idoneidad del instrumento utilizado.

La coherencia macroscópico-microscópico, asociada a la categoría II, aparece lograda estrictamente en gas y solo aceptable en UNLP (figs. 1 y 3), donde los porcentajes ron-

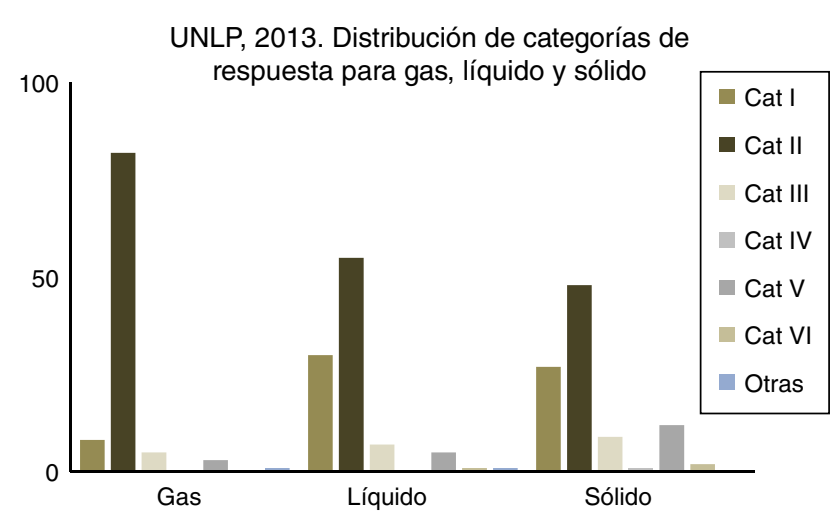

Figura 3 Distribución de respuestas para gas, líquido y sólido en estudiantes de UNLP (Física I, curso 2013).

UNLP: Universidad Nacional de La Plata. 


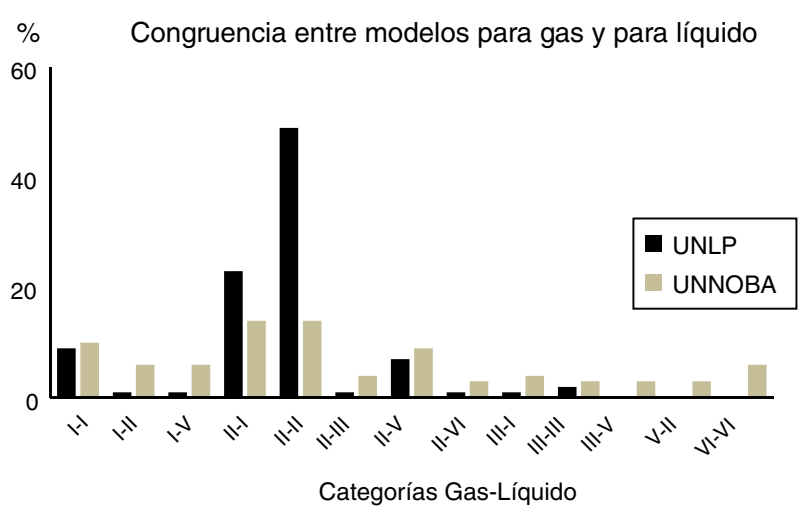

Figura 4 Congruencias gas-líquido. Distribución de respuestas de estudiantes de UNLP y UNNOBA, cursos 2007.

UNLP: Universidad Nacional de La Plata; UNNOBA: Universidad Nacional del Noroeste de la Provincia de Buenos Aires.

dan el $80 \%$. En esta universidad, sin embargo, no surge un modelo dominante para líquido y sólido repartiéndose entre las categorías I y II y con menor presencia de las restantes. Los resultados de UNNOBA reflejan una situación aún peor: la categoría II domina en gas con solo un $46 \%$, pero la I resulta importante (con un 21\%) y crece al pasar a líquido para prevalecer en sólidos con el $46 \%$, es decir, al mismo nivel que la II para gas.

\section{Sobre congruencia en la utilización de modelos microscópicos en las 3 fases}

Las figuras 4-7 se refieren al uso de un único modelo para los distintos estados de agregación y comparan los resultados obtenidos en los cursos 2007 de ambas unidades académicas. Lo que evidencian es que en UNLP, para las fases tomadas de a pares, resulta dominante la conjunción ॥-॥ acompañada por algunas más. En cambio, en UNNOBA se nota una marcada dispersión de respuestas (cabe acotar que el orden en las conjunciones indicadas alude al orden de fases de la congruencia correspondiente; así la conjunción II-v en la

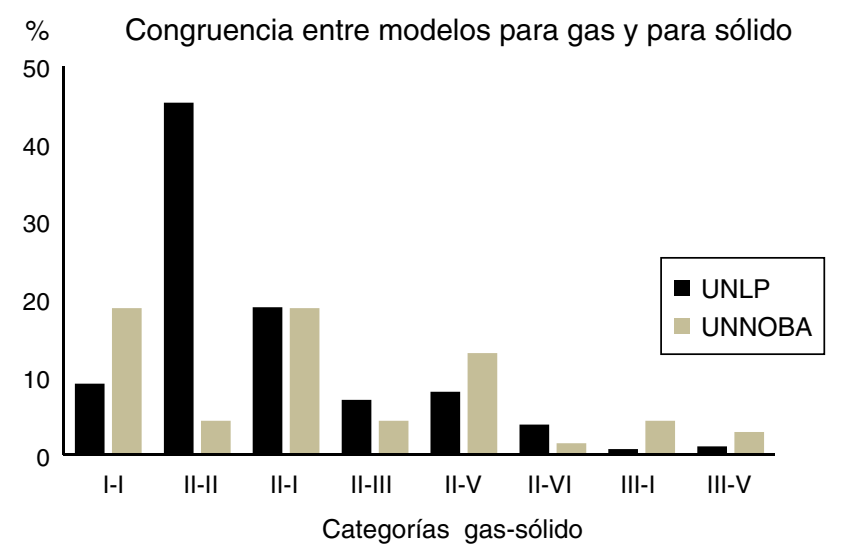

Figura 5 Congruencias gas-sólido. Distribución de respuestas de estudiantes de UNLP y UNNOBA, cursos 2007.

UNLP: Universidad Nacional de La Plata; UNNOBA: Universidad Nacional del Noroeste de la Provincia de Buenos Aires.

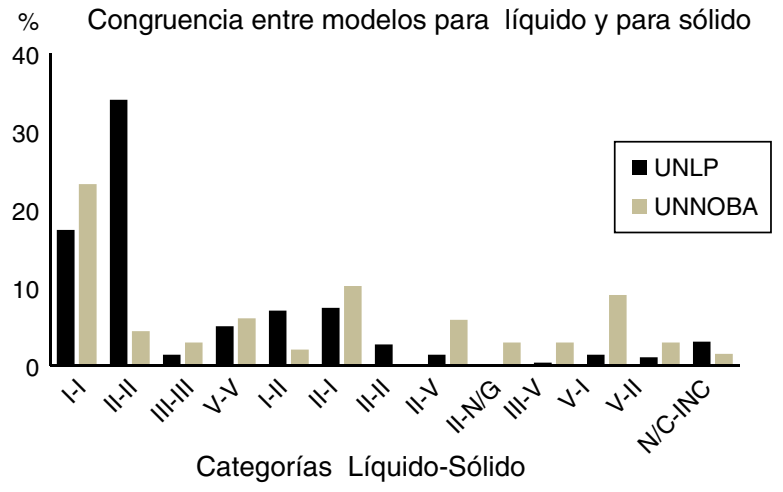

Figura 6 Congruencias líquido-sólido. Distribución de respuestas de estudiantes de UNLP y UNNOBA, cursos 2007.

UNLP: Universidad Nacional de La Plata; UNNOBA: Universidad Nacional del Noroeste de la Provincia de Buenos Aires.

figura 4, por ejemplo, indica la categoría I para gas y la v para líquido).

Aun así, en UNLP los porcentajes obtenidos para II-I resultan bajos si se espera una respuesta compatible con la concepción de la comunidad científica después de un año de Química universitaria: un 49\% para gas-líquido en la figura 4, un 45\% para gas-sólido en la figura 5 y un 34\% para líquido-sólido en la figura 6. En UNNOBA, esta conjunción deseable alcanza muy bajos porcentajes, con un $14 \%$ para gas-líquido y un $4 \%$ tanto para líquido-sólido como para gassólido. Además, la figura 4 (congruencia gas-líquido) indica que se destacan las conjunciones II-I (con el 23\% para UNLP y el $14 \%$ para UNNOBA), I-I (con el $9 \%$ para UNLP y el $10 \%$ para UNNOBA) y II-v (con el 7\% para UNLP y el 9\% para UNNOBA), aunque menores que la ॥-I. Que la conjunción II-I resulte importante refleja que domina una representación discreta aunque en líquido no se acepta la compresión, es decir, se mantiene la afirmación persistente desde la escolaridad primaria de la incompresibilidad de líquidos aun cuando el enunciado de la encuesta propone que «[... el el espacio ocupado por el agua disminuya [...]». Esto se ve afianzado ya que las conjunciones que siguen en porcentaje son la I-ly la II-v (esta última indica que, para líquido, aparece una representación no discreta).

En la figura 5 aparece la distribución de frecuencias para congruencia entre gas y sólido. Nuevamente, la II-II aparece como mayoritaria en UNLP (con el $45 \%$ ), a la que le siguen

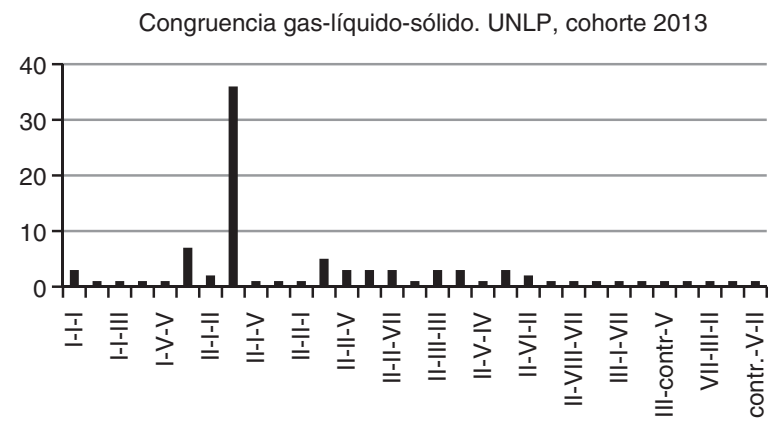

Figura 7 Congruencias gas-líquido-sólido. Distribución de respuestas de estudiantes de UNLP, 2013.

UNLP: Universidad Nacional de La Plata. 
las conjunciones II-I (con el 19\%) y ו- (con el 9\%). Las diferencias con UNNOBA resultan mayúsculas ya que, en esta universidad, la conjunción II-II solo alcanza el 4\%. Las más significativas en UNNOBA resultan la II-I, la I-I (ambas con el $19 \%$ ) y la II-v (con el 13\%). Nuevamente, el perfil de respuestas de UNNOBA aparece como disperso frente a lo obtenido en UNLP. Desde el punto de vista de los modelos dominantes para cada fase se puede afirmar que, al igual que para líquido, en sólido se manifiesta como dominante un modelo discreto pero que no muestra coherencia con lo fenomenológico (categoría ı) para ambas universidades. En menor medida y para UNNOBA solamente, aparece la categoría $v$ que no incluye una representación discreta.

La figura 6 presenta la distribución de respuestas para la congruencia líquido-sólido. En UNLP aparecen como conjunciones dominantes la II-II (con el 34\%) y la I-I (con el 17\%). Para UNNOBA la situación es a la inversa: la conjunción de mayor porcentaje es la I-I (con el 23\%), mientras que la IIII se reduce a un $4 \%$. La siguiente conjunción notable es la II-I, con el $7 \%$ para UNLP y el $10 \%$ para UNNOBA. En esta última universidad se distingue la v-ı (con el 9\%), mientras que esa conjunción resulta muy minoritaria para UNLP (con el 1\%). Esta figura confirma lo expuesto en las anteriores respecto de las representaciones para estas fases, ya que las categorías principales son la $\|$, la I y la $v$, aunque se registra un fuerte sesgo hacia la I (sobre todo en UNNOBA), es decir, hacia un modelo discreto sin coherencia con lo macroscópico.

La figura 7 muestra los resultados para UNLP, cohorte 2013, respecto de la congruencia para gas-líquido-sólido. Se observa la dominancia de la combinación $\|-\|-\|$ con un $36 \%$. La única otra combinación notable, con un $7 \%$, es la II-I-I, lo cual implica que solo para gas aparece la respuesta deseada, mientras que para líquido y para sólido se elige la categoría I indicativa de la existencia de una representación discreta sin coherencia con lo observable. Estos resultados están muy lejos de lo deseable: solo el $36 \%$ de los estudiantes interpretan las situaciones planteadas desde una única representación acorde con el modelo establecido por la comunidad científica. Es llamativo que en ambas universidades aparezca un resultado importante para la categoría II en gas y un notable sesgo hacia la categoría I en líquido y sólido. Esto podría desvirtuar una posible falla en la interpretación de la encuesta en alguna de las 2 universidades. Un aspecto a considerar sería el referido al término «partícula». La polisemia de esa palabra puede asociarla a átomos, moléculas o a trozos pequeños de sustancia. Sin embargo, la elección de opciones para los 3 casos considerados en la encuesta no se vería afectada por uno u otro de los contenidos del término, además de que es la palabra utilizada en la teoría cinética expuesta durante el ciclo secundario. Precisamente, consideramos que la presentación de esa teoría en el marco de gases ideales probablemente constituya una influencia mayor en las respuestas obtenidas, evidenciando la escasa utilización de este tipo de modelizaciones en líquidos y sólidos a lo largo del sistema educativo (Guevara y valdez, 2004).

\section{Consideraciones finales}

Si bien el instrumento utilizado reviste un carácter exploratorio y limitado por las opciones propuestas (no permite evaluar si la representación microscópica del encuestado incluye movimiento de las partículas constituyentes), los resultados reflejan perfiles, en ambas instituciones académicas, en los que solo en el caso de gas aparece una modelización compatible con la pretendida desde el conocimiento científico para encuestados que recorrieron, al menos, un año de Química universitaria.

Con relación a la coherencia en el uso del modelo asociado a la teoría de partículas, se evidencian inconvenientes para interpretar propiedades macroscópicas de líquidos y sólidos, acudiéndose a una diversidad de representaciones y mostrando, en muchos casos, una contradicción entre la representación y lo fenomenológico y aun dentro mismo del modelo discreto.

En cuanto a las congruencias gas-líquido, gas-sólido y líquido-sólido, aparecen dificultades notables: en UNLP hay una baja elección de la combinación deseable ॥-II, en tanto que en UNNOBA se refleja una gran dispersión en las opciones elegidas. La congruencia entre las 3 fases refleja porcentajes aún menores de la combinación deseable (figura 7). La pintura global estaría indicando que las representaciones microscópicas discretas no son mayoritarias y dominan en el estado gaseoso. Es decir, la articulación entre los conocimientos generados desde el ámbito científico y las representaciones de cada estudiante no es la deseada. Esto enfatiza la necesidad de extender, a lo largo del sistema educativo, el uso del modelo discreto para los 3 estados de agregación en el abordaje de fenómenos que los incluyan.

Resulta llamativo el peso de la categoría I, indicando una representación discreta, aunque incompatible con lo fenomenológico, para líquido y sólido. El enunciado en la encuesta marca el cambio de volumen de la sustancia ante la presión ejercida sobre el émbolo. La negación de lo señalado reflejaría la preponderancia de afirmaciones absolutas emitidas desde el saber escolar (dureza de sólidos e incompresibilidad de líquidos, más allá de cualquier contexto) y que se combinan de forma desconocida con el saber propio (de origen vivencial) constituyendo un híbrido inestable pero persistente (Taber y García-Franco, 2010). La importancia de esta categoría en ambas instituciones refuerza la necesidad de indagar sobre estas cuestiones entrevistando a estudiantes y docentes y considerando diversos trabajos de aula.

Coincidiendo con otros trabajos (Giudice y Galagovsky, 2008; García-Franco y Taber, 2010; Taber y García-Franco, 2010; Adbo y Taber, 2014) consideramos que las dificultades evidenciadas en estudiantes universitarios después de un año de Química, ante situaciones muy simples, constituyen elementos importantes en la reformulación del trabajo en el aula de ciencias para todos los niveles educativos. La persistente dificultad en la articulación entre lo macroscópico y lo submicroscópico acentúa la necesidad de replantear el recorrido de aprendizaje y exige una mayor preocupación por el trabajo en clase sobre modelización en Química y sus limitaciones en relación con diferentes sustancias, fases y contextos. 


\section{Conflicto de intereses}

\section{Los autores declaran no tener ningún conflicto de intereses.}

\section{Referencias}

Adbo, K. y Taber, K. (2014). Developing an understanding of chemistry: A case study of one Swedish student's rich conceptualisation for making sense of upper secondary school chemistry. International Journal of Science Education, 36(7), 1107-1136.

Atkins, P. y Jones, L. (1998). Química: moléculas, materia y cambio (3. ${ }^{\mathrm{a}}$ ed.). Barcelona: Ediciones Omega.

Chang, R. (1995). Química (4. ${ }^{\mathrm{a}}$ ed.). México: Mc Graw-Hill.

Cooper, M., Corley, L. y Underwood, S. (2013). An investigation of college chemistry students' understanding of structure-property relationships. Journal of Research in Science Teaching, 50(6), 699-721.

Duschl, R., Hamilton, R. y Grandy, R. E. (1990). Psychology and epistemology: Match or mismatch when applied to science education? International Journal of Science Education, 27(3), 367-383.

Ebenezer, J. y Fraser, D. (2001). First year chemical engineering students' conceptions of energy in solution processes: Phenomenographic categories for common knowledge construction. Science Education, 85(5), 509-535.

Espíndola, C. y Cappannini, O. (2012). Obstáculos en la evaluación diagnóstica. Una propuesta de superación mediante la identificación de modelos presentes en el curso. Educación Química, 23(4), 484-491.

Flores-Camacho, F., Gallegos-Cázares, L., Garritz, A. y GarcíaFranco, A. (2007). Incommensurability and multiple models: Representations of the structure of matter in undergraduate chemistry students. Science \& Education, 16(7-8), 775-800.

García-Franco, A. y Taber, K. (2010). Pensamiento intuitivo y aprendizaje de la química. Educación Química, 21(2), 111-117.

Giudice, J. y Galagovsky, L. (2008). Modelar la naturaleza discontinua de la materia: una propuesta para la Escuela Media. Revista Electrónica de Enseñanza de las Ciencias, 7(3), 629-657.

Glasstone, S. (1970). Tratado de química física ( $7 .^{\mathrm{a}} \mathrm{ed}$.). Madrid: Editorial Aguilar.
Guevara, M. y Valdez, R. (2004). Los modelos en la enseñanza de la Química: algunas de las dificultades asociadas a su enseñanza y a su aprendizaje. Educación Química, 15(3), 243-247.

Hammer, D. (2004). The variability of student reasoning. En E. Redish y M. Vicentini (Eds.), Proceedings of the Enrico Fermi Summer School, Course CLVI. Italia: Bologna [consultado 15 Dic 2014]. Disponible en: http://dhammer.phy.tufts.edu/home/ publications_files/Hammer\%20Varenna\%203.preprint.pdf

Johnson-Laird, P. (1983). Mental models: Towards a cognitive science of language inference and consciousness. Cambridge, MA: Harvard University Press.

Johnstone, A. H. (2010). You can't get there from here. Journal of Chemical Education, 87(1), 22-29.

Krnel, D., Watson, R. y Glazar, S. A. (2005). The development of the concept of 'matter': A cross-age study of how children describe materials. International Journal of Science Education, 27(3), 367-383.

Linder, C. y Marshall, D. (2003). Reflection and phenomenography: Towards theoretical and educational development possibilities. Learning and Instruction, 13, 271-284.

Norman, D. N. (1983). Some observations on mental models. En D. Gentner y A. L. Stevens (Eds.), Mental models (pp. 7-14). Hillsdale, NJ: Erlbaum.

Pozo, J. I. y Rodrigo, M. J. (2001). Del cambio de contenido al cambio representacional en el conocimiento conceptual. Infancia y Aprendizaje, 24(4), 407-423.

Stains, M. y Talanquer, V. (2007). Classification of chemical substances using particulate representations of matter: An analysis of student thinking. International Journal of Science Education, 29(2), 643-661.

Taber, K. y García-Franco, A. (2010). Learning processes in chemistry: Drawing upon cognitive resources to learn about the particulate structure of matter. Journal of the Learning Sciences, 19, 99-142.

Taber, K. y Watts, M. (1996). An explanatory gestalt of essence: Students' conceptions of the 'natural' in physical phenomena. International Journal of Science Education, 18(8), 939-954. 